\title{
Preferential host-guest coordination of nonactin with ammonium and hydroxylammonium
}

Cite as: J. Chem. Phys. 149, 225101 (2018); https://doi.org/10.1063/1.5049956

Submitted: 26 July 2018 . Accepted: 20 November 2018 . Published Online: 11 December 2018

Bruno Martínez-Haya (D), Juan Ramón Avilés-Moreno, Francisco Gámez (D), Giel Berden, and Jos Oomens

\section{ARTICLES YOU MAY BE INTERESTED IN}

Effect of the chemical environment of the DNA guanine quadruplex on the free energy of binding of $\mathrm{Na}$ and $\mathrm{K}$ ions

The Journal of Chemical Physics 149, 225102 (2018); https://doi.org/10.1063/1.5050534

Granularity impact on hotspot formation and local chemistry in shocked nanostructured RDX

The Journal of Chemical Physics 149, 224703 (2018); https://doi.org/10.1063/1.5049474

Impact of cross-linking of polymers on transport of salt and water in polyelectrolyte membranes: A mesoscopic simulation study

The Journal of Chemical Physics 149, 224902 (2018); https://doi.org/10.1063/1.5057708

The Journal 


\title{
Preferential host-guest coordination of nonactin with ammonium and hydroxylammonium
}

\author{
Bruno Martínez-Haya, ${ }^{1, a)}$ Juan Ramón Avilés-Moreno, ${ }^{1}$ Francisco Gámez, ${ }^{1}$ \\ Giel Berden, ${ }^{2}$ and Jos Oomens ${ }^{2}$ \\ ${ }^{1}$ Department of Physical, Chemical and Natural Systems, Universidad Pablo de Olavide, \\ 41013 Seville, Spain \\ ${ }^{2}$ Radboud University, Institute for Molecules and Materials, FELIX Laboratory, Toernooiveld $7 c$, \\ 6525ED Nijmegen, The Netherlands
}

(Received 26 July 2018; accepted 20 November 2018; published online 11 December 2018)

\begin{abstract}
The biological activity of the macrocycle nonactin is intimately related to its ionophore properties and ability to act as a selective cation carrier. The competitive binding of small protonated amines constitutes a particularly key issue in the biochemistry of nonactin, which finds application in sensing and extraction technologies. In this study, isolated complexes of nonactin with ammonium and hydroxylammonium are investigated with infrared action spectroscopy and quantum chemical computations. The focus of the investigation is on the coordination achieved by the protonated guest with the oxygen atoms of either the oxolane groups or the carboxyl groups in the ester linkages of the macrocyle host and their relative contributions to the stability of the complexes. The experimental and computational data converge to a preferred coordination arrangement associated with a tight binding of the $\mathrm{N}-\mathrm{H}^{\delta+}$ bonds with the oxolane groups. In the $\mathrm{NH}_{4}^{+}$complex, this results in a compact complex of $S_{4}$ symmetry. In contrast, symmetry is disrupted in the $\mathrm{NH}_{3} \mathrm{OH}^{+}$complex, as it incorporates a bifurcated coordination of the $-\mathrm{OH}$ bond with a carbonyl group and an oxolane group of the host, involving also a more stretched arrangement of the nonactin backbone. These gas-phase conformations are in agreement with the structures postulated for these complexes in condensed phases, from previous Raman and crystallographic experiments. Published by AIP Publishing. https://doi.org/10.1063/1.5049956
\end{abstract}

\section{INTRODUCTION}

Nonactin is a naturally occurring macrotetrolide with a recognized potential as carrier of metal cations, ammonium, and protonated amines. ${ }^{1-3}$ The oxygen atoms from the oxolane (tetrahydrofuran) and ester linkages of the macrocycle backbone (see Fig. 1) promote the encapsulation of the cation and the outer exposure of a hydrophobic surface. Nonactin complexes can therefore incorporate guest cations into organic phases and constitute fundamental benchmark systems for the regulation of ionic transport through the lipid bilayers of cell membranes, leading to selective antibiotic action. ${ }^{4,5}$ The salient ionophoric features of nonactin have inspired the synthesis of a considerable number of model compounds aimed at specific transport or sensing applications in biotechnology. In particular, the outstanding affinity of nonactin for $\mathrm{NH}_{4}^{+}$has motivated the development of a range of molecular materials for its recognition and recovery from industrial and biological products, the performance of each novel material being systematically contrasted to that of nonactin as reference. ${ }^{6-10}$

The early pioneering studies of the supramolecular chemistry of nonactin already established the large ammonium

a)Author to whom correspondence should be addressed: bmarhay@upo.es affinity of this macrocycle in crystallographic x-ray diffraction $^{11,12}$ and Raman vibrational spectroscopy ${ }^{13}$ measurements, which was attributed to a tight coordination of the charged $\mathrm{N}-\mathrm{H}^{\delta+}$ bonds of ammonium with the ether oxygen atoms of the oxolane rings of nonactin. This scenario may change for less symmetric and larger secondary amines, whose coordination arrangements are likely to involve a more active role of the oxygen atoms from the ester carbonyl groups of the macrocycle. Hydroxylammonium, the model compound of choice for this study, incorporates a simple polar $-\mathrm{OH}$ substituent with respect to ammonium. In addition to the physiological, environmental, and technological importance of hydroxylamine, its molecular structure conforms an excellent benchmark to pinpoint the ability of nonactin to adopt stretched non-symmetric conformations in order to cope with the multipodal coordination and steric effects imposed by guest molecular ions.

This study combines mass spectrometry and laser action spectroscopy with quantum chemical modelling, to obtain insights into the dominant conformations and coordination structures that sustain the complexes formed by nonactin with ammonium and hydroxylammonium. Similar recent investigations have served to assess fine conformational aspects of the selective complexation of alkali cations by nonactin, with a detailed evaluation of the influence of hydration. ${ }^{14,15}$ In this work, we extend the scope of the investigation to 


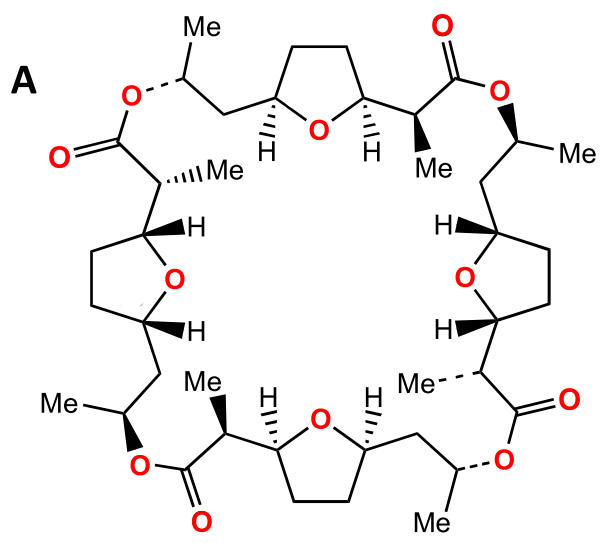

B
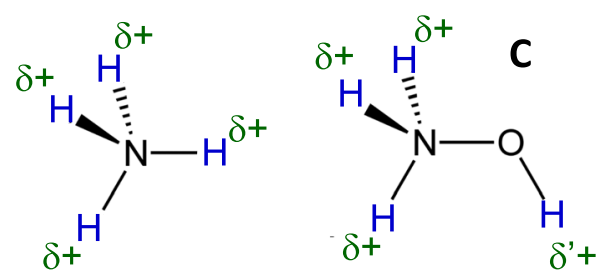

FIG. 1. Schematic representation of the host ionophore nonactin [(a), with $\left.\mathrm{Me}=\mathrm{CH}_{3}\right]$ and of the two guest protonated amines investigated in this work, ammonium (b) and hydroxylammonium (c).

protonated amine guests, as a first incursion into a line of research in our group aimed at the fundamental understanding of the recognition of amines of increasing complexity by nonactinic materials.

\section{MATERIALS AND METHODS}

\section{A. Infrared multiple photon dissociation spectroscopy}

Infrared multiple photon dissociation (IRMPD) spectra were recorded using a Fourier transform ion cyclotron resonance (FT-ICR) mass spectrometer, coupled to the infrared beamline of the laser facility FELIX (Free Electron Laser for Infrared Experiments). ${ }^{16,17}$ The cationic complexes were produced via electrospray ionization (ESI) of a water/methanol (1:1) solution $0.1 \mathrm{mM}$ in nonactin and $1 \mathrm{mM}$ in ammonium chloride or hydroxylammonium chloride, with added acetic acid to help avoid deprotonation of the amine cation. Nonactin from Streptomyces griseus ( $>98 \%$ purity) and the chloride salts ( $>99 \%$ purity) were purchased from SigmaAldrich and used without further treatment. The positive ions are produced in a Waters Z-spray ESI source (with the needle voltage set at $+2.7 \mathrm{kV}$ ), accumulated in a hexapole ion trap $\left(10^{-3}\right.$ Torr $)$, and subsequently pulse-injected into the ICR cell for mass isolation The mass-selected ions are irradiated with $\sim 10-20$ FELIX macro-pulses of $5 \mu$ s duration and $\sim 35 \mathrm{~mJ}$ energy. Each macro-pulse consists of trains of micropulses with a repetition rate of $1 \mathrm{GHz}$. The spectral bandwidth of the radiation amounts to $0.5 \%$ of the central wavelength, which in this study was scanned between $900 \mathrm{~cm}^{-1}$ and $1800 \mathrm{~cm}^{-1}$.

The resonant absorption of multiple photons by the trapped ions at wavenumbers associated with specific vibrational modes leads to fragmentation. The IRMPD spectrum is obtained by monitoring the total yield of ionic fragments, with linear corrections to account for changes in laser pulse energy during the frequency scans. The main fragments observed in our experiments have masses $m / z=737,553,369$, and 185 and are identified as protonated oligomers of the form $\left(\mathrm{C}_{10} \mathrm{H}_{16} \mathrm{O}_{3}\right)_{n} \cdot \mathrm{H}^{+}, n=4$, corresponding to the full nonactin macrocycle. Weaker signals from fragments resulting from additional $\mathrm{H}_{2} \mathrm{O}$ loss were also observed. For the $\mathrm{NH}_{4}^{+}$complex, the intensity of the fragments decreased as $n=2>3>1$ $>>4$, whereas for the $\mathrm{NH}_{3} \mathrm{OH}^{+}$complex, the intensity trend was $n=4>2>3>1$. Hence, the main fragmentation product of the $\mathrm{NH}_{3} \mathrm{OH}^{+}$complex (protonated nonactin, $n=4$ ) was not appreciable for the $\mathrm{NH}_{4}^{+}$complex. It seems timely to remark that the analogous fragments observed in a previous IRMPD study of the alkali cation complexes of nonactin followed the similar trend $\left(\mathrm{C}_{10} \mathrm{H}_{16} \mathrm{O}_{3}\right)_{n} \cdot \mathrm{M}^{+}$, with $n=2,3\left(\mathrm{M}^{+}\right.$representing the alkali cation). ${ }^{15}$

\section{B. Determination of relative stabilities of the complexes}

Electrospray ionization mass spectrometry was also employed to assess the relative stabilities of the complexes of nonactin with $\mathrm{NH}_{4}^{+}, \mathrm{NH}_{3} \mathrm{OH}^{+}$, and $\mathrm{K}^{+}$. In this case, the measurements were performed in a Q-Exactive Focus Orbitrap Mass Spectrometer (Thermo-Fisher). Methanol solutions $10 \mu \mathrm{M}$ in nonactin and $200 \mu \mathrm{M}$ in chlorides of ammonium, hydroxylammonium, and potassium were sprayed at a needle voltage of $3.8 \mathrm{kV}$. The mass spectrum was recorded at a resolution of $\mathrm{M} / \Delta \mathrm{M}=70000$ and displayed neat signals from the three cation complexes as described below.

\section{Quantum chemical calculations}

Density functional theory (DFT) computations were employed to characterize the most stable conformations of the isolated complexes of nonactin with ammonium and hydroxylammonium. The procedure started with a survey of the conformational landscape of the complexes performed with simulated annealing, combining the predictions of well known force fields (Universal, Dreiding). The approximately forty non-redundant conformations of lowest energy were optimized in B3LYP/6-31+G(d) computations. Finally, the resulting twenty most stable conformations were reoptimized at the B3LYP/6-311++G(d,p) level. The conformers have been ranked throughout this study according to their zero-point corrected electronic energies. Natural bond orbital (NBO) theory ${ }^{18}$ was employed for a detailed assessment of the proton bonding interactions that sustain the complexation process. The IR spectrum of each conformer was built from the convolution of the predicted fundamental mode frequencies and intensities with a Gaussian line broadening of $20 \mathrm{~cm}^{-1}$ (full width at half maximum). The computed harmonic vibrational frequencies were scaled by a factor 0.98 in comparison with the experiment, in line with standard recommendations. ${ }^{19}$

The role of anharmonicities in the vibrational spectrum was studied for the most stable conformer of the $\mathrm{NH}_{4}^{+}$complex. The full anharmonic treatment of a system of the size of the nonactin complexes was not affordable. Therefore, a restricted mode computation was performed, in which anharmonicity 
was incorporated to a selected ensemble of fundamental modes particularly relevant in the comparison of the computational predictions with the IRMPD experiment. ${ }^{20}$ Specifically, for this study, the effective anharmonic transition frequencies of the three main modes related to umbrella vibrational motions of the $\mathrm{NH}_{4}^{+}$guest were computed within the secondorder vibrational perturbation approximation $(\mathrm{VPT} 2)^{21}$ at the B3LYP/6-311++G(d,p) level, while the rest of modes were kept harmonic. This approach is based on the strategies outlined in seminal methodologies for the modelling of large molecular systems ${ }^{22-24}$ and has been applied as implemented in the Gaussian 09 suite of programs. ${ }^{25}$

\section{RESULTS}

The quantum chemical survey of the ammonium and hydroxylammonium complexes of nonactin converged to the most stable conformations that are depicted in Fig. 2. The ammonium complex will be discussed in the first place, as it constitutes a consolidated benchmark for the ionophore

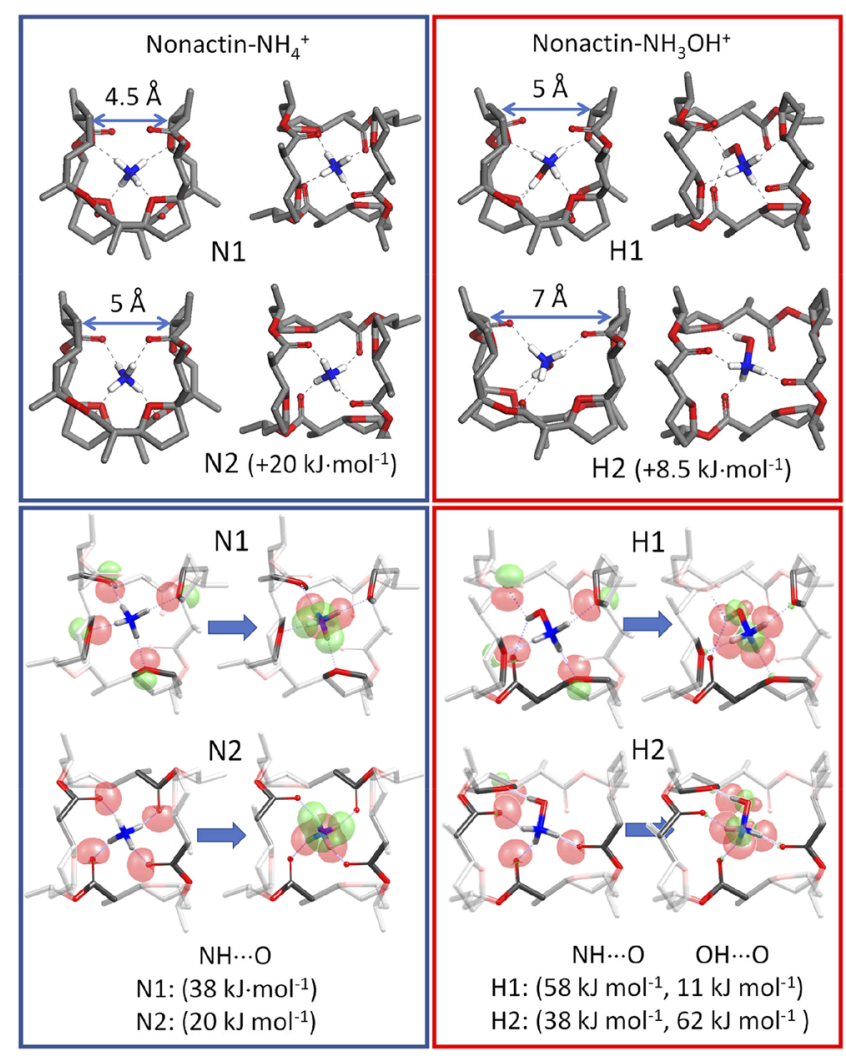

FIG. 2. Top panels: Top and side views of the two most stable conformations of the complexes of nonactin with the cations $\mathrm{NH}_{4}^{+}(\mathbf{N 1}$ and $\mathbf{N 2})$ and $\mathrm{NH}_{3} \mathrm{OH}^{+}$ (H1 and H2). The difference in zero-point corrected electronic energy between the conformers is indicated. Note that conformers N1 and H1 involve coordination of the ammonium moieties with the oxygen atoms in the oxolane (tetrahydrofuran) groups of nonactin, whereas in $\mathbf{N 2}$ and $\mathbf{H 2}$, the coordination takes place with the carbonyl groups of the ester bonds. Bottom panels: Natural bond orbitals responsible for the redistribution of charge that sustains the stability of each conformation. Transfer of electronic charge occurs from the lone pairs of the oxygen atoms of nonactin to the antibonding $\sigma^{*}$ orbitals of the $-\mathrm{NH}^{\delta+}$ and $-\mathrm{OH}$ bonds of the guest cations, leading to the average stabilization energies per bond indicated in the lower part of each panel. behavior of nonactin and should guide the rationalization of the recognition of small protonated amines by this macrocycle.

\section{A. Nonactin- $\mathrm{NH}_{4}^{+}$complex}

The ammonium complex displays two low energy conformations of $\mathrm{S}_{4}$ symmetry, both of which share a similar arrangement of the nonactin backbone. The two conformers are essentially connected by a rotation of the $\mathrm{NH}_{4}^{+}$cation replacing the coordination with the oxygen atoms of the four oxolane groups in the most stable conformer N1 with those of the four carbonyl groups in the conformer next in energy, $\mathbf{N} 2$. The H-bonding NH . . O distances are similar in the two conformations (1.9 $\AA$ ). This implies a somewhat greater stretching of the nonactin backbone in conformer $\mathbf{N} 2$ in comparison to $\mathbf{N 1}$, in order to accommodate to the optimum coordination distance between the cation and the inward-oriented carbonyl groups. Figure 2 shows that the opening of the cage-like conformation adopted by nonactin is $\sim 0.5 \AA$ larger in N2 versus N1.

The B3LYP computation yields as much as $20 \mathrm{~kJ} \mathrm{~mol}^{-1}$ energy difference between conformers N1 and N2. Higher energy conformers were found at relative energies at least $40 \mathrm{~kJ} \mathrm{~mol}^{-1}$ above N1, featuring less compact coordination arrangements associated with more open and asymmetric structures of the nonactin backbone. Conformer N1 is therefore predicted to account for the dominant conformation of the nonactin- $\mathrm{NH}_{4}^{+}$complex under the present experimental conditions. The structural features of conformer $\mathbf{N 1}$ are in fact in excellent agreement with the crystal structure determined for the $\mathrm{NH}_{4} \mathrm{NCS}$ complex of nonactin, which resulted in a $\mathrm{S}_{4}$ conformation with a $\mathrm{N}$... O distance in the oxolane coordination moiety of $2.9 \AA,{ }^{11}$ which is coincident with the one obtained for the N1 conformer of the isolated complex in the present B3LYP computation.

Natural bond orbital analysis provides further structural and energetic insights into the relevance of the competitive $\mathrm{H}$ bonding attained by $\mathrm{NH}_{4}^{+}$inside the nonactin host. In the $\mathbf{N 1}$ conformer, the $\mathrm{H}$-bonds are formed at $45^{\circ}$ from the COC plane of the oxolane groups, while in the $\mathbf{N} 2$ conformer, they lie at $60^{\circ}$ from the ester carbonyl plane. Otherwise, both types of coordination arrangements feature roughly collinear $\mathrm{H}$ bonds, with $\mathrm{NH} \cdots \mathrm{O}$ angles $\sim 160^{\circ}-170^{\circ}$. Figure 2 highlights the main donor and acceptor orbitals that sustain the host-guest coordination in each case. The transfer of electronic charge occurs from the lone pairs of the oxygen atoms in the oxolane or ester carbonyl groups towards the antibonding $\sigma^{*}$ orbitals of the $\mathrm{NH}^{\delta+}$ bonds of ammonium. The stabilization energy associated with the charge transfer from the oxolane groups in conformer $\mathbf{N 1}\left(38 \mathrm{~kJ} \mathrm{~mol}^{-1}\right.$ per bond) is almost a factor of two larger than that from the carbonyl groups in conformer $\mathbf{N} 2\left(20 \mathrm{~kJ} \mathrm{~mol}^{-1}\right)$, which serves to explain the greater stability of oxolane coordination.

It is interesting to notice that the directional tetradentate coordination that drives the recognition of ammonium by nonactin is in contrast with the scenario associated with s-shell metal cation complexes. Previous studies have shown that the complexes of nonactin with alkali and alkaline earth cations 
adopt a $\mathrm{S}_{4}$ configuration similar to the one found for $\mathrm{NH}_{4}^{+}$. However, the spherical symmetry of the atomic guest yields an effective electrostatic eight-fold coordination with all the oxolane and carbonyl groups at $\mathrm{M}^{+} \ldots \mathrm{O}$ distances ranging from 2.8-3.0 $\AA$ for $\mathrm{K}^{+}$to $3.2-3.3 \AA$ for $\mathrm{Cs}^{+}{ }^{+}{ }^{13,15}$ The NBO analysis leads in this case to stabilization energies of similar magnitude in the coordination of the alkali cation with the oxolane and carbonyl groups, e.g., of $\sim 15 \mathrm{~kJ} \mathrm{~mol}^{-1}$ per bond for $\mathrm{K}^{+}$.

The vibrational signatures of the nonactin- $\mathrm{NH}_{4}^{+}$complex should serve to assess the accuracy of the computational predictions described above. Figure 3 shows the IRMPD spectrum recorded for this complex, along with the IR spectra associated with conformers N1 and N2. A good overall agreement is found between computation and experiment for the structure of the vibrational spectrum, which supports the reliability of the technique for the structural characterization of nonactin-amine complexes. A rich progression of vibrational bands is observed within the range of wavenumbers $900-1800 \mathrm{~cm}^{-1}$ scoped in these experiments. The main band structures are labelled $A-G$ for discussion and are qualitatively assigned to normal vibrational modes of the complex as described in Table I. It can be noted that the ammonium-carbonyl coordination induces a significant coupling of the vibrational motions of the nonactin backbone with the bending modes of the $\mathrm{NH}_{4}^{+}$moiety (or $\mathrm{NH}_{3}^{+}$ in hydroxylamine).

It is particularly interesting to note that the coordination of the carbonyl groups in conformer $\mathbf{N} 2$ induces a red shift of the $\mathrm{C}=\mathrm{O}$ stretching band (band A) by $\sim 35 \mathrm{~cm}^{-1}$ with respect to conformer N1. This is illustrated in Fig. 3 in terms of the relative position between band $\mathrm{A}$ and band $\mathrm{E}$ in the IR spectra computed for the two conformers. Note that band $\mathrm{E}$ is associated with nonactin backbone vibrations $(\mathrm{C}-\mathrm{O}-\mathrm{C}$ stretching of the ester bonds and $\mathrm{C}-\mathrm{C}$ stretching in the oxolane rings) that appear to be weakly affected by the precise $\mathrm{NH}_{4}^{+}$coordination arrangement attained in the complex, as long as the conformation of the macrocycle stays roughly unchanged as it is the case. The position of band $\mathrm{A}$ in the experimental IRMPD spectrum $\left(1711 \mathrm{~cm}^{-1}\right.$, versus $1198 \mathrm{~cm}^{-1}$ for band E) supports then a situation of weakly interacting carbonyl groups and, hence, the coordination of ammonium with the oxolane groups predicted by the computation for conformer N1.

Despite the good overall agreement between the IRMPD measurement and the computational IR spectrum of conformer $\mathbf{N 1}$, there is one outstanding discrepancy for one particular spectral feature. The B3LYP computation predicts a pronounced vibrational band at around $1500 \mathrm{~cm}^{-1}$ for which no apparent trace is found in the experiment. The three vibrational transitions that contribute to this band are highlighted with red colour bars in Fig. 3 and are assigned by the computation to umbrella modes of the $\mathrm{NH}_{4}^{+}$cation, with a virtually static nonactin host. Previous studies have shown that bending modes in ammonium and protonated primary amines are likely to display anharmonic behavior. ${ }^{26}$ In order to explore this possibility for the present complexes, we performed an additional B3LYP computation for the $\mathbf{N} 1$ conformer in which the frequencies of the three umbrella modes of ammonium were treated anharmonically within the VPT2 approximation, while the remaining vibrational modes of the complex were kept harmonic. Given the size of nonactin, a full anharmonic computation of the complex was not possible with our computational resources. The partial anharmonic treatment applied here must be interpreted as a preliminary approximation to the modelling of the complex useful to assess possible trends inferred by anharmonicity in its spectral signatures. ${ }^{20,22-24}$ Figure 3 shows that the result of the anharmonic computation is a red shift of the three modes by $\sim 50 \mathrm{~cm}^{-1}$. Such a shift merges these modes with band $\mathrm{B}$, thereby reconciling the qualitative structure of the computational IR bands with the one displayed

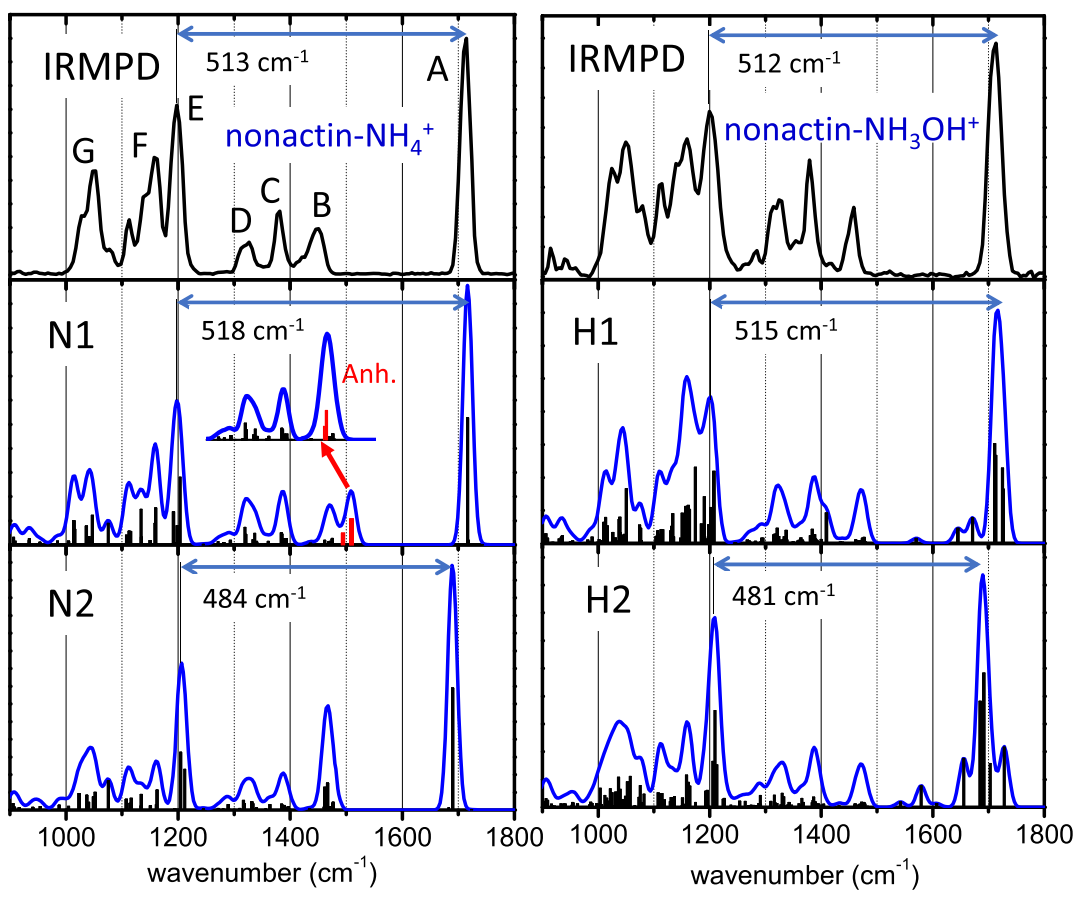

FIG. 3. IRMPD spectra of the complexes of nonactin with $\mathrm{NH}_{4}^{+}$and $\mathrm{NH}_{3} \mathrm{OH}^{+}$(top panels), and the IR spectra predicted by the B3LYP/6$311++\mathrm{G}(\mathrm{d}, \mathrm{p})$ computations for the corresponding conformers of lowest energy (middle and bottom panels). See Fig. 2 for a representation of the conformers. The main vibrational bands, labelled A-G for discussion, are described qualitatively in Table I. The position of band A with respect to band $\mathrm{E}$ is indicated as a signature of the potential participation of the ester carbonyl bonds in the coordination with the guest cation. The bar diagrams in the computational spectra represent the position and IR intensity of each vibrational transition. The bars in red colour for the N1 conformer highlight the vibrational modes associated with umbrella vibrations of the $\mathrm{NH}_{4}^{+}$cation. The inset shows the spectrum resulting from the treatment of these modes within a partial anharmonic approximation (see the text). 
TABLE I. Vibrational mode assignment for the main bands observed in the IRMPD spectra of the complexes of nonactin with ammonium $(\mathrm{N})$ and hydroxylamine $(\mathrm{H})$ (see labels $\mathrm{A}-\mathrm{G}$ in Fig. 3). The dominant types of vibrational motions are indicated in each case.

\begin{tabular}{|c|c|c|}
\hline Band & Range $\left(\mathrm{cm}^{-1}\right)$ & Mode assignment \\
\hline A & $1695-1730$ & $\begin{array}{c}\mathrm{C}=\mathrm{O} \text { stretching }(\mathrm{str} .) \\
\mathrm{NH}_{4}^{+} \text {bending }(\mathrm{N}) \\
\mathrm{NH}_{3}^{+} \text {bending/umbrella }(\mathrm{H})\end{array}$ \\
\hline B & $1410-1480$ & $\begin{array}{c}\mathrm{CH}_{2} / \mathrm{CH}_{3} \text { scissoring } \\
\mathrm{NH}_{4}^{+} \text {umbrella }(\mathrm{N})\end{array}$ \\
\hline $\mathrm{C}$ & $1360-1400$ & $\begin{array}{c}\mathrm{CH} / \mathrm{CH}_{2} \text { wagging, } \mathrm{CH}_{3} \text { umbrella } \\
\mathrm{NOH} \text { bending }(\mathrm{H})\end{array}$ \\
\hline $\mathrm{D}$ & $1300-1345$ & $\mathrm{CH}_{2}$ rocking/wagging \\
\hline $\mathrm{E}$ & $1180-1225$ & $\mathrm{COC} / \mathrm{CC}$ str., $\mathrm{CH}_{2}$ twisting \\
\hline \multirow[t]{2}{*}{$\mathrm{F}$} & $1100-1180$ & $\mathrm{COC} / \mathrm{CC}$ str., $\mathrm{CH}_{2}$ twisting, \\
\hline & & $\mathrm{CH}_{3}$ rocking \\
\hline G & $1010-1090$ & $\begin{array}{c}\mathrm{CO} \text { str. (oxolane), } \mathrm{CH}_{2} \text { rocking } \\
\mathrm{N}-\mathrm{O} \operatorname{str}(\mathrm{H})\end{array}$ \\
\hline
\end{tabular}

by the IRMPD spectrum in the $1300-1500 \mathrm{~cm}^{-1}$ range. In summary, the anharmonic treatment corroborates the finding that conformer N1 constitutes the configuration of the nonactin$\mathrm{NH}_{4}^{+}$complex that is dominantly populated at ambient temperature.

\section{B. Nonactin- $\mathrm{NH}_{3} \mathrm{OH}^{+}$complex}

The hydroxylammonium guest introduces a differentiated coordination, involving specific H-bonding and steric interactions of its $\mathrm{OH}$ group, in addition to those of the $\mathrm{NH}^{\delta+}$ bonds. As a result, this complex does not display the symmetry found for the ammonium complex, and mixed oxolane/carbonyl coordination arrangements become feasible. Figure 2 depicts the two most stable conformations for the hydroxylamine complex of nonactin. The conformation of lowest energy, H1, features H-bonding of the three ammonium bonds with oxolane groups of nonactin (at NH . . O distances 1.80-1.85 and angles $160^{\circ}-180^{\circ}$ ), while the $\mathrm{OH}$ group displays a bifurcated coordination with the oxygen atoms from a carbonyl group (at $2.05 \AA$ and $140^{\circ}$ ) and from the fourth oxolane group (at $2.04 \AA$ and $114^{\circ}$ ). The situation reverses in the H2 conformer, in which the ammonium moiety of hydroxylamine coordinates with three carbonyl groups of nonactin (at 1.75-1.80 $\AA$ and $155^{\circ}-165^{\circ}$ ), while the $\mathrm{OH}$ groups bind to an oxolane group (at $1.70 \AA$ and $175^{\circ}$ ). The B3LYP computation assigns an energy difference, between conformers $\mathbf{H 1}$ and $\mathbf{H 2}$, of $8.5 \mathrm{~kJ} \mathrm{~mol}^{-1}$. Figure 2 shows that the coordination of hydroxylammonium with the carbonyl groups in conformer $\mathbf{H 2}$ stretches the nonactin backbone, leading to a cavity opening as much as $\sim 2 \AA$ larger than the one found for conformer $\mathbf{H 1}$.

The NBO analysis for the $\mathrm{NH}_{3} \mathrm{OH}^{+}$complex corroborates the trend found for the $\mathrm{NH}_{4}^{+}$complex. The $\mathrm{NH}^{\delta+} \ldots \mathrm{O}$ coordination of the three ammonium bonds with the oxolane groups of the host in conformer H1 leads to a much larger stabilization energy (58 $\mathrm{kJ} \mathrm{mol}^{-1}$ per bond in average) than the coordination with the carbonyl groups in conformer $\mathbf{H 2}$ ( $38 \mathrm{~kJ} \mathrm{~mol}^{-1}$ per bond). Interestingly, the $\mathbf{H} \mathbf{2}$ conformer displays a strong $\mathrm{OH} \cdots \mathrm{O}$ interaction $\left(62 \mathrm{~kJ} \mathrm{~mol}^{-1}\right)$ between the $\mathrm{OH}$ bond of hydroxylamine and one oxolane group of nonactin. In comparison, the bifurcated interaction of the $\mathrm{OH}$ bond with the oxolane and carbonyl groups amounts to only 9 and $13 \mathrm{~kJ} \mathrm{~mol}^{-1}$, respectively. This finding serves to rationalize the fact that the relative energy of the $\mathbf{H} \mathbf{2} / \mathbf{H 1}$ conformers $\left(8.5 \mathrm{~kJ} \mathrm{~mol}^{-1}\right)$ is more than a factor of two smaller than the one found for $\mathbf{N} 2 / \mathbf{N 1}\left(20 \mathrm{~kJ} \mathrm{~mol}^{-1}\right)$.

The IRMPD spectrum recorded for the nonactin$\mathrm{NH}_{3} \mathrm{OH}^{+}$complex is represented in Fig. 3. The analysis of the corresponding IR spectra predicted for conformers $\mathbf{H} 1$ and $\mathbf{H} 2$ leads to a rationalization of the experiment in similar terms as outlined above for the $\mathrm{NH}_{4}^{+}$complex. Hydroxylammonium incorporates $\mathrm{NOH}$ bending and $\mathrm{N}-\mathrm{O}$ stretching vibrational modes to bands $\mathrm{F}$ and $\mathrm{G}$, respectively. Coupling of $\mathrm{C}=\mathrm{O}$ stretching and $\mathrm{NH}_{3}^{+}$bending modes is also observed in band $\mathrm{A}$ for conformer $\mathbf{H 2}$. The vibrational band structure associated with conformer $\mathbf{H 1}$ resembles closely the IRMPD measurement throughout the full spectral range investigated. In particular, the position of the $\mathrm{C}=\mathrm{O}$ stretching band is consistent with that found in the IRMPD spectrum. Although not obvious at first sight, in the IRMPD spectrum, the $\mathrm{C}=\mathrm{O}$ stretching band broadens slightly towards its red flank, with respect to that of the $\mathrm{NH}_{4}^{+}$complex. This is interpreted in terms of a red shift by $\sim 10 \mathrm{~cm}^{-1}$ of two modes associated with the motions of the single $\mathrm{C}=\mathrm{O}$ group that is $\mathrm{H}$-bonded to the $\mathrm{OH}$ group of the guest cation in conformer H1. In contrast, it can be noted that in the $\mathbf{H} \mathbf{2}$ conformer, the three coordinated $\mathrm{C}=\mathrm{O}$ bonds lead to a splitted stretching band whose main component is clearly red shifted with respect to the experiment. The present experimental and computational results hence suggest that conformer $\mathbf{H 1}$ is the only arrangement of the complex that is significantly populated at ambient temperature.

\section{Relative complex stabilities}

It is interesting to notice that, whereas the nonactin$\mathrm{NH}_{4}^{+}$complexes could be readily characterized with crystallographic techniques, ${ }^{11}$ this has not been possible for the $\mathrm{NH}_{3} \mathrm{OH}^{+}$complexes. Attempts to form nonactin- $\mathrm{NH}_{3} \mathrm{OHCl}$ crystals have been reported, which failed presumably due to an unfavorable balance of solvent and crystal field interactions. ${ }^{13}$ In order to shed some light into this finding, we close the study with an assessment of the relative stability of the nonactin complexes of ammonium and hydroxylammonium. For this purpose, electrospray ionization measurements were performed on methanol solutions equimolar in $\mathrm{NH}_{4}^{+}, \mathrm{NH}_{3} \mathrm{OH}^{+}$, and $\mathrm{K}^{+}$, all of them in excess with respect to nonactin (see Sec. II). The inclusion of $\mathrm{K}^{+}$provides a valuable benchmark and serves to estimate the potential competitive binding of both species in the presence of common alkali metal cations. The resulting mass spectrum is shown in Fig. 4 and reflects the relative abundances of the three complexes. It can be observed that the signal detected for the $\mathrm{NH}_{4}^{+}$complex is four-fold and 


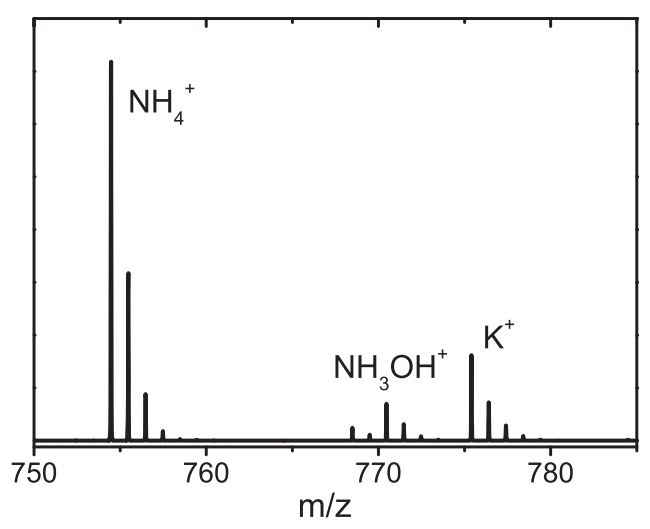

FIG. 4. Electrospray ionization mass spectrum of a methanol solution equimolar in $\mathrm{NH}_{4}^{+}, \mathrm{NH}_{3} \mathrm{OH}^{+}$, and $\mathrm{K}^{+}(200 \mu \mathrm{M})$, in excess with respect to nonactin $(10 \mu \mathrm{M})$. The ratios of the collected ion signals are of $\mathrm{NH}_{4}^{+}: \mathrm{NH}_{3} \mathrm{OH}^{+}: \mathrm{K}^{+}$ of 1:0.12:0.25 and reflect the relative abundances of the complexes of nonactin with the three cations in the solution.

eight-fold more intense than the signals associated with the $\mathrm{K}^{+}$ and $\mathrm{NH}_{3} \mathrm{OH}^{+}$complexes, respectively. Hence, the binding of ammonium by nonactin can be expected to be robust under a broad range of environmental conditions, whereas the stabilization of the hydroxylammonium complex can plausibly be more sensitive to the presence of other cations and to solvent interactions.

\section{CONCLUSIONS}

The infrared spectra of isolated nonactin complexes with $\mathrm{NH}_{4}^{+}$and $\mathrm{NH}_{3} \mathrm{OH}^{+}$have been characterized with IRMPD action spectroscopy and modelled by means of DFT quantum chemical calculations. The most stable computational structures for the two complexes (conformers N1 and H1) involve tight coordination arrangements of the ammonium moieties with the oxolane ether oxygen of the nonactin host. In the ammonium complex, a particularly compact conformation of $\mathrm{S}_{4}$ symmetry is attained. In the hydroxylammonium complex, such symmetry is disrupted, as the $\mathrm{OH}$ group of the guest cation forms a complementary bifurcated H-bond with the fourth oxolane group and a carbonyl group of the macrocycle. The computational structures next in energy for the two complexes (N2 and H2) feature complementary coordination arrangements of ammonium with carbonyl groups of the macrocycle and involve a more stretched conformation of the nonactin backbone.

Natural bond orbital analysis rationalizes the stability of the two nonactin complexes in terms of an efficient distribution of charge from the lone pair orbitals of the oxolane ether oxygen atoms of the macrocycle to the antibonding orbitals of the $\mathrm{NH}^{\delta+}$ bonds in the ammonium moieties of the guest cations. The stabilization energies from such interactions are roughly a factor of two stronger than those associated with the carbonyl oxygen atoms from the ester groups of the macrocycle. The bifurcated coordination of the $\mathrm{OH}$ group of hydroxylammonium contributes more moderately to the stability of the complex in the $\mathbf{H 1}$ conformation.

The vibrational band structure observed in the IRMPD spectrum supports the preferred coordination of ammonium with the oxolane ether oxygen atoms of nonactin, in particular, the position of the $\mathrm{C}=\mathrm{O}$ stretching bands in the spectra of the two complexes is consistent with conformers $\mathbf{N 1}$ and $\mathbf{H 1}$ and rules out any significant population of higher energy conformers involving significant ammoniumcarbonyl interactions. These results corroborate earlier assignments in condensed phases from Raman and crystallography measurements.

Anharmonicity appears to play a relevant role in the bending modes of the ammonium guest moieties in the complexes. The partial VPT2 treatment, restricted to these modes, appears to constitute a fair approximation to the modelling of these systems. Future work in our group will seek further challenges to modelling approaches by considering complexes of nonactin with protonated amine guests of increasing complexity, including the important case of amino acids.

\section{ACKNOWLEDGMENTS}

The research leading to these results has received funding from Junta de Andalucia-FEDER (Project No. P12-FQM2310) and from LASERLAB-EUROPE (Grant Agreement No. 654148, EU Horizon 2020 research and innovation programme). We thank C3UPO for the HPC support. We gratefully acknowledge the Nederlandse Organisatie voor Wetenschappelijk Onderzoek (NWO) for the support of the FELIX Laboratory.

${ }^{1}$ W. Keller-Schlierlein and H. Gerlach, Fortschr. Chem. Org. Naturst. 26, 161 (1968).

${ }^{2}$ A. Samat, M. E. M. Bibout, J. Chanon, and J. Elguero, New J. Chem. 6, 483 (1982).

${ }^{3}$ A. Samat, M. E. M. Bibout, and J. Elguero, J. Chem. Soc., Perkin Trans. 1 8, 1717 (1985).

${ }^{4}$ Z. Zizka, Folia Microbiol. 43, 7 (1998).

${ }^{5}$ L.-J. Ming, Med. Res. Rev. 23, 697 (2003).

${ }^{6}$ K. Suzuki, D. Siswanta, T. Otsuka, T. Amano, T. Ikeda, H. Hisamoto, R. Yoshihara, and S. Ohba, Anal. Chem. 72, 2200 (2000).

${ }^{7}$ J. Chin, J. Oh, S. Y. Jon, S. H. Park, C. Walsdorff, B. Stranix, A. Ghoussoub, S. J. Lee, H. J. Chung, S. M. Park, and K. Kim, J. Am. Chem. Soc. 124, 5374 (2002).

${ }^{8}$ H. Y. Jin, T. H. Kim, J. Kim, S. S. Lee, and J. S. Kim, Bull. Korean Chem. Soc. 25, 59 (2004).

${ }^{9}$ R. Carrillo, V. S. Martín, M. López, and T. Martín, Tetrahedron 61, 8177 (2005).

${ }^{10}$ A. Späth and B. König, Beilstein J. Org. Chem. 6, 32 (2010).

${ }^{11}$ K. Neupert-Laves and M. Dobler, Helv. Chim. Acta 59, 614 (1976).

${ }^{12}$ Y. Nawata, T. Sakamaki, and Y. Iitaka, Acta Crystallogr., Sect. B: Struct. Crystallogr. Cryst. Chem. 33, 1201 (1977).

${ }^{13}$ I. M. Asher, G. D. J. Phillies, B. J. Kim, and H. E. Stanley, Biopolymers 16, 157 (1977).

${ }^{14}$ B. Martínez-Haya, J. R. Avilés-Moreno, S. Hamad, and J. Elguero, Phys. Chem. Chem. Phys. 19, 1288 (2017).

${ }^{15}$ J. R. Avilés-Moreno, F. Gámez, G. Berden, J. Oomens, and B. MartínezHaya, Phys. Chem. Chem. Phys. 19, 14984 (2017).

${ }^{16}$ N. C. Polfer and J. Oomens, Mass Spectrom. Rev. 28, 468 (2009).

${ }^{17} \mathrm{See}$ http://www.ru.nl/felix/ for more information on the FTICR mass spectrometer coupled to the FELIX beam line and for the specifications of the FELIX infrared radiation.

${ }^{18}$ J. P. Foster and F. Weinhold, J. Am. Chem. Soc. 102, 7211 (1980).

${ }^{19}$ NIST Standard Reference Database 101, Computational Chemistry Comparison and Benchmark DataBase (Release 18 October 2016), http://cccbdb.nist.gov/vibscalejust.asp

${ }^{20}$ J. R. Avilés-Moreno, G. Berden, J. Oomens, and B. Martínez-Haya, Phys. Chem. Chem. Phys. 20, 8968 (2018).

${ }^{21}$ V. Barone, J. Chem. Phys. 122, 014108 (2005). 
${ }^{22}$ V. Barone, M. Biczysko, J. Bloino, M. Borkowska-Panek, I. Carnimeo, and P. Panek, Int. J. Quantum Chem. 112, 2185 (2012).

${ }^{23}$ M. Piccardo, J. Bloino, and V. Barone, Int. J. Quantum Chem. 115, 948 (2015).

${ }^{24}$ J. Bloino and M. Biczysko, Int. J. Quantum Chem. 116, 1543 (2016).

${ }^{25}$ M. J. Frisch, G. W. Trucks, H. B. Schlegel, G. E. Scuseria, M. A. Robb, J. R. Cheeseman, G. Scalmani, V. Barone, B. Mennucci, G. A. Petersson, H. Nakatsuji, M. Caricato, X. Li, H. P. Hratchian, A. F. Izmaylov, J. Bloino, G. Zheng, J. L. Sonnenberg, M. Hada, M. Ehara, K. Toyota, R. Fukuda, J. Hasegawa, M. Ishida, T. Nakajima, Y. Honda, O. Kitao, H. Nakai, T. Vreven, J. A. Montgomery, Jr., J. E. Peralta, F. Ogliaro, M. Bearpark,
J. J. Heyd, E. Brothers, K. N. Kudin, V. N. Staroverov, R. Kobayashi, J. Normand, K. Raghavachari, A. Rendell, J. C. Burant, S. S. Iyengar, J. Tomasi, M. Cossi, N. Rega, J. M. Millam, M. Klene, J. E. Knox, J. B. Cross, V. Bakken, C. Adamo, J. Jaramillo, R. Gomperts, R. E. Stratmann, O. Yazyev, A. J. Austin, R. Cammi, C. Pomelli, J. W. Ochterski, R. L. Martin, K. Morokuma, V. G. Zakrzewski, G. A. Voth, P. Salvador, J. J. Dannenberg, S. Dapprich, A. D. Daniels, Ö. Farkas, J. B. Foresman, J. V. Ortiz, J. Cioslowski, D. J. Fox, Gaussian 09, Revision E.01, Gaussian, Inc., Wallingford, CT, 2009.

${ }^{26}$ P. Hurtado, F. Gámez, S. Hamad, B. Martínez-Haya, J. D. Steill, and Oomens, J. Phys. Chem. A 115, 7275 (2011). 